\title{
Design of Effective Smart Communication System for Impaired People
}

\author{
Dr. Akey Sungheetha \\ Data Science SIG member, \\ Image Processing SIG member, \\ Computer Science and Engineering, \\ School of Electrical Engineering and Computing, \\ Adama Science and Technology University, \\ Adama, Nazret, \\ Ethiopia. \\ Dr. Rajesh Sharma R \\ Image Processing SIG member, \\ Computer Science and Engineering, \\ School of Electrical Engineering and Computing, \\ Adama Science and Technology University, \\ Adama, Nazret, \\ Ethiopia.
}

Abstract- In communication medium, sharing a conversation dialogue between the normal person and deaf and dumb person is one of the challenging tasks still. The dumb person can practice hand gesture language in their community but not to others. This research article focuses to minimize the difficulty level between these two communities with smart glove devices. Besides, the author believes that result of the proposed model provides a good impact on the dump community. The smart glove contains input, control, and output module to get, process, and display the data respectively. Our proposed model is used to help these communities to interact with each other continuously without any error. The proposed model is constructed with good specification flex sensors. Little change of resistance in flex sensor is providing changes in their gesture language. So this orientation direction is calculated well and gives better results over existing methods. The wireless set can be made with Bluetooth technologies here. Here the gestures are assigned based on the alphabet letter. The sign language performs and gives audible output in the display section of the proposed model. It gives good results in our experimental setup. This research work focuses on good recognition rate, accuracy, and efficiency. The good recognition rate shows the continuous 
Journal of Electrical Engineering and Automation (EEA) (2020)

Vol. 02/ No. 04

Pages: 181-194

https://www.irojournals.com/iroeea

DOI: https://doi.org/10.36548/jeea.2020.4.006

conversation between the two persons. Moreover, this research article compares the recognition rate, accuracy, and efficiency of the proposed model with an existing model.

Keywords: Gesture recognition system, Flex sensor, Gesture language

\section{INTRODUCTION}

According to a survey, 2.42M people are deaf and dumb people in India that large amount in the society. Overall the world is having around $15-20 \%$ of the deaf and dumb population [1]. This community is facing communication problems in society and the neighboring area. This communication gap is creating lots of problems and isolation of a particular community. The common person communication constituent is not sufficient for all other communities [2]. The term "Augmentative and Alternative Communication" refers to speech components other than audible type communication. It consists of many symbols, figures, rising tones of synthesized speech, and drawing with sign language. Augmentative model communication comprises various datasets for limited abilities of their speech [3].

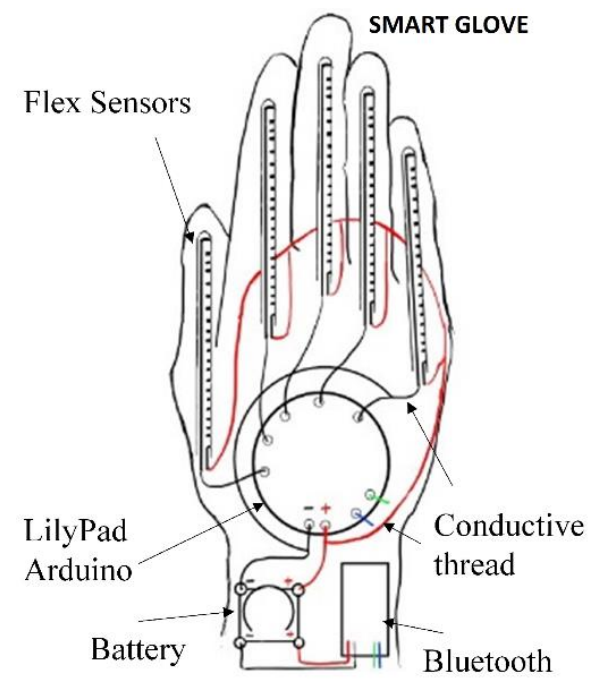

Figure 1 Overview structure of Smart Glove

Figure 1 shows smart glove pictures with flex sensors, Arduino, battery, conductivity thread, and a transmitter section. This mode of communication is supporting both people in the community. Generally, the alternative model is comprised of the database for their corresponding sign and symbols [4]. No speech persons will interpret their idea, desire, needs to the normal person. The 
Journal of Electrical Engineering and Automation (EEA) (2020)

Vol. 02/ No. 04

Pages: 181-194

https://www.irojournals.com/iroeea

DOI: https://doi.org/10.36548/jeea.2020.4.006

communication systems are customized for many specific situations such as a person visit doctor, a person attends an interview, and so on. Also, the communication devices are controlled by many central processing units of the computer or laptop [5][6][7]. The communication devices are not easy to carry and handle in all the places where they want. The electronic system should be easy to handle and portable, dedicated for the corresponding person [8][9][10]. The electronic system can be classified into two as follows;

1. Dedicated electronics system

2. Undedicated electronics system

The dedicated device is used to do communication between the person that speaking by electronic devices. The undedicated devices are including speaking with many sign functions and feature extraction which will function with the central processing unit of the devices [11]. It can provide internet facilities, e-mail services, etc. Our proposed system contains both of the operations lead by electronic devices. Figure 2 shows the components used in our project.

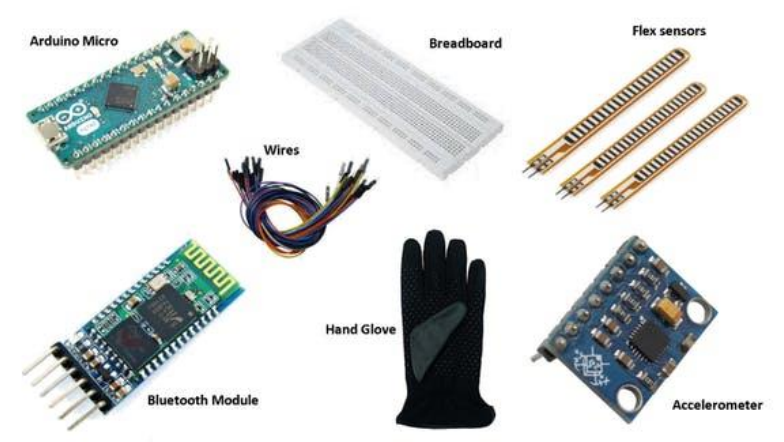

Figure 2 Components used for our project

The dumb peoples are having lack communication with normal and deaf and dumb people's communities [12]. Also, they are facing language problems of various regional problems in India. So there is a need for a translator for both of them which is not easy in all circumstances [13][14][15]. The application model should be considered those parameters and unique interpreters commonly [16]. Sign language is a varying person to person for communication. And our audio signal should be synchronized for pre-determined with our data. This will accomplish an essential 
Journal of Electrical Engineering and Automation (EEA) (2020)

Vol. 02/ No. 04

Pages: 181-194

https://www.irojournals.com/iroeea

DOI: https://doi.org/10.36548/jeea.2020.4.006

task for normal and deaf and dumb society [17] [18]. Figure 3 shows the flex sensor circuit diagram.

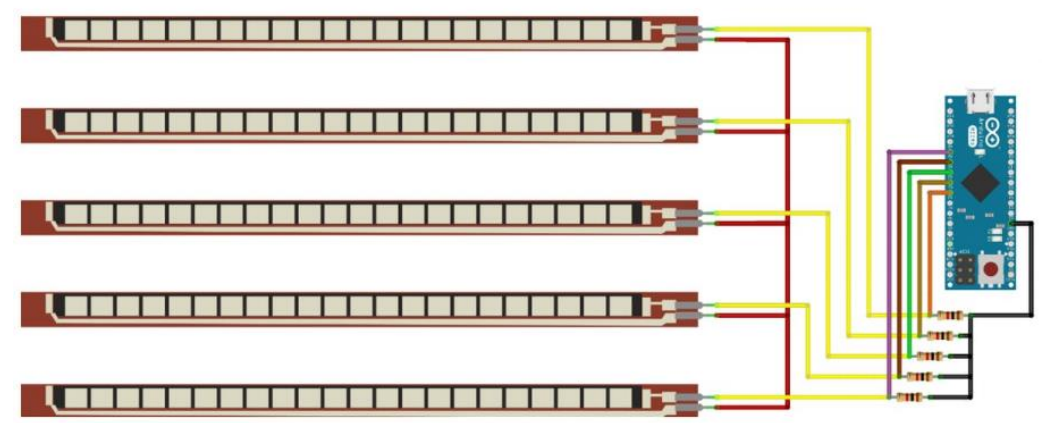

Figure 3 Circuit diagram of flex sensor integration

\section{Motivation}

The deaf and dumb person faces many problems in interaction with others, education sectors, mental health with low safety measures of them. There is an absence of a communication medium between them still. According to many recent research surveys, the physical disability is inattentive between deaf and dumb people with a normal person. The disabled person's confidence level is very poor due to the absence of communication devices. And sign language is very difficult to learn and remember by a normal person. The development of smart gloves is providing some recreation in the human community.

\section{ORGANIZATION OF THE RESEARCH}

The structure of the research article is organized as follows; Section 3 gives existing recent research papers on augmentative communication methods. Section 4 discusses the methodology of our proposed approach, Section 5 delivers discussion about results obtained by our proposed model. The conclusion and the future task discusses in section 6 .

\section{PRELIMINARIES}

M.P. Paulraj et al proposed a machine vision system with Artificial Neural Network (ANN). They developed a sign hand gesture recognition system with nine phonemes in a foreign language. They constructed a model consists of the input stage, classification stage, display stage. The input stages are consisting of detection of color and region of interest segmentation which is done by image registration method [19]. M. Mohandes et al. introduced Hidden Markov Model 
Journal of Electrical Engineering and Automation (EEA) (2020)

Vol. 02/ No. 04

Pages: 181-194

https://www.irojournals.com/iroeea

DOI: https://doi.org/10.36548/jeea.2020.4.006

(HMM) for image classification. They proposed an image-based detection signer's face. The Gaussian skin model has been developed for this detection and each frame of the images is tracking by a region-based method. The recognition stage is computed from feature extraction from given images for the process [20]. Wang et al proposed a tensor subspace analysis for hand gesture analysis. They developed a model for multi-view hand gestures for a better recognition process. This sign language recognition system is accomplishing over and done with image segmentation. They developed RGB color image detection by a backpropagation network model. They checked with a cross-validation process for matching the input hand gesture images [21]. Kuo Chue Neo et al is implemented a sign translation system to detect hand gesture sign language from the input images. The images are captured by camera here to do preprocessing before the detection process. The numerical representation is used to identify the gesture posture from the input images. They implemented an FPGA chip with a core process to construct a model for proposing a system. They experimented with image process ideas and it is improving the speed of the processes [22]. K. Park et al proposed a gesture recognition system for sign identification by Field Programmable Gate Array (FPGA). They developed a smart glove as the prototype for a real-time environment. They concentrated on some problems aroused during the process like space, lighting, and motion problems in real-time environmental conditions. They constructed a system with main three modules called preprocessing, controlling, and display module. Their proposing model recognizes the hand gesture by various identification functions of input group data and it is comparing with existing methods. The comparison process is the very pre-defined signal of hand gestures and they are displayed in the Liquid Crystal Display (LCD) screen [23]. W.K. Chung et al introduce the wavelet transform method in the recognition system. They implemented many schemes for realtime hand gesture identification. The codeword scheme is one of the effective methods to identify the hand gesture for the matching process. Their proposed system reduces the database size by removing unwanted features from principal storage. They contain a digital camera for capturing the hand gesture from input data. They consider in their process that transforms technique with "Haar" wavelets [24].

The skin color also will be detected by this RGB value of feature extraction classification in every single frame in an image. The controlling stage consists of many feature extraction and detection units themselves. The moment invariant feature can be extracted and computed by many 
Journal of Electrical Engineering and Automation (EEA) (2020)

Vol. 02/ No. 04

Pages: 181-194

https://www.irojournals.com/iroeea

DOI: https://doi.org/10.36548/jeea.2020.4.006

sets of images from the motion of the signer's hand gesture images. This classification stage is performed by ANN to recognize the gesture motion. They computed the average recognition rate is $92.85 \%$ [19]. Tushar Chouhan et al constructs a smart glove system that contains many sensors to detect the hand gesture of the person standing opposite of him. They contain many sections in their proposed system. They developed a system consist of many bend sensors for hand sign language which consist of a flexible tube, a photodiode is used to capture the light and infrared diode. The steerable orientation property of the hand sign can be detected by an accelerometer. They developed with the co-ordinate axis in various positions. The multiplexer unit is used to integrate many bend sensors and its output is taken by them. The output of the multiplexer provides anyone of the action to avoid all the sensor works at the same time in the circuit. This integrated circuit consists of a converter circuit that is converting from current to voltage phenomena. This analog portion should convert from that domain to digital by Analog to Digital Converter (ADC) in the circuit which is used for integrating the with a smart glove. The digital devices can be incorporated with an analog circuit through the ADC element. The machine learning method is used to help tune the hand gesture motion based on the user's comfort [12]. The understandable form can be derived from the raw input images based on their past attempts the system can be trained. The previous attempts can be carried out for the next iteration process to implement the smart glove with a machine learning algorithm [6].

\section{METHODOLOGIES}

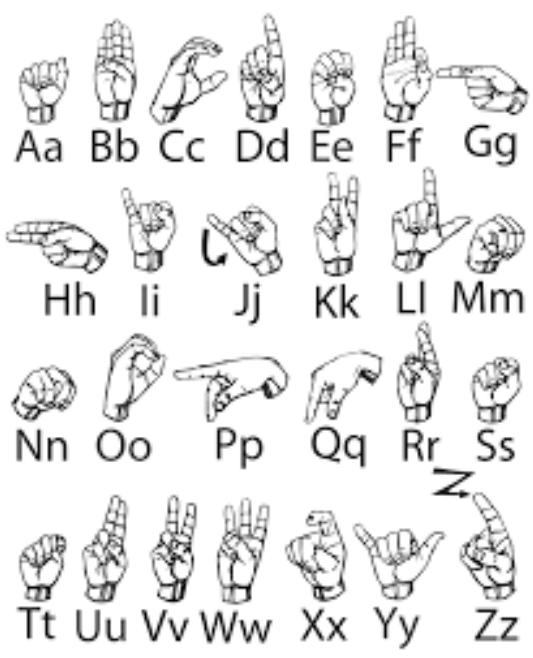

Figure 4 Standard hand sign from disabled person 
Journal of Electrical Engineering and Automation (EEA) (2020)

Vol. 02/ No. 04

Pages: 181-194

https://www.irojournals.com/iroeea

DOI: https://doi.org/10.36548/jeea.2020.4.006

This paper aims to construct the smart glove to convert sign language to the audible speech signal. Our proposed system is implementing for capturing the images from the disabled person and identify the hand gesture him [18]. The standard hand sign from the dumb person is showing in figure 4. The gloves are designing for identifying the motion of the hand gesture. The smart gloves consist of many bend sensors which are used to support convert some electrical signal to data signal as speech [25]. The motion of a hand gesture is given to a smart glove which consists of a microcontroller unit within it for the further process of a model. The gesture signal is transmitting through Bluetooth for communication medium. The recognized gesture is matching with pre-defined data and it is providing to the display unit or speaker unit for voice recognition. Our proposed model has two sections as transmitter and receiver [26]. Figure 5 shows the process flow of the transmitter section of the proposed method.

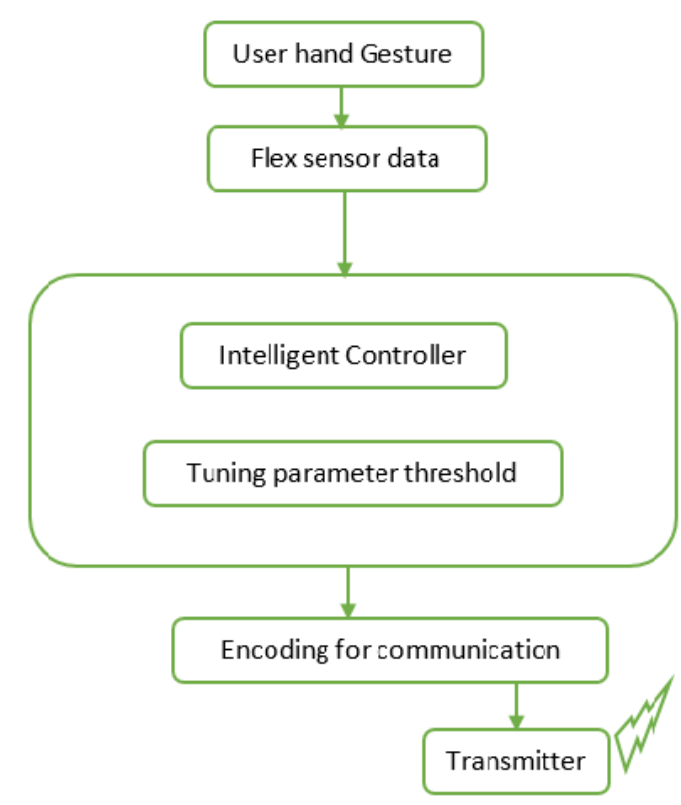

Figure 5 The process flow of transmitter section of proposed method

Figure 6 shows the workflow of the receiver section of the proposed method. The additional gesture recognition system provides a better understanding between the people and improving the accuracy, recognition rate, and efficiency of the system compared with existing methods [27]. 


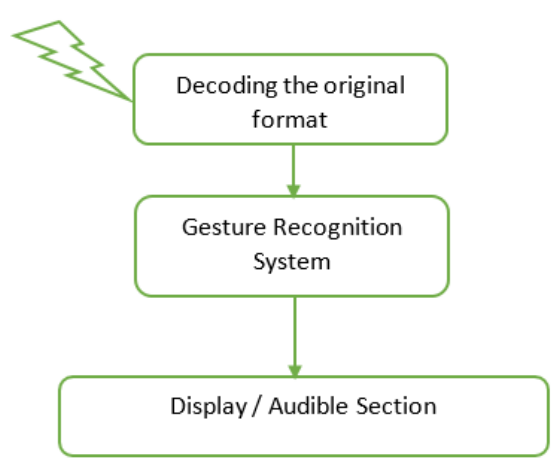

Figure 6 The process flow of receiver section of proposed method

Other existing methods are microcontroller unit alone provided less recognition rate. Our proposed method gives a better recognition rate which is shown in the graph in figure 8 . There is an essential development for communication between the deaf person with a normal person [28]. The design of smart gloves is used to identification of sign language and to communicate between them. The intelligent system microcontroller is used to develop a model that contains many sections for getting, controlling, displaying the data. The low-level coding can be written for the controller unit to control the sensor action in the smart glove based on the gesture movement [29]. The recognition is used to identify and convert it to an audible speech signal which is already stored in a storage. Due to cost-effectiveness, the system is constructed with a microcontroller unit with codes [30][31]. The normal person will type the text as the message that is converted into sign language to display the deaf person. This arrangement will provide more comfortable to both persons due to this process is vice versa. This communication is the wireless transmission (Bluetooth) of the data between devices [32][33][34].

\section{RESULTS DISCUSSION}

The different resistance values of flex sensor with various degree of sensors position. Based on these flex sensors in our proposed model gives a more appropriate answer for the hand gesture compared to many bend sensors output. This appropriate answer is making the comfort zone between both the persons in confidently. The confidence level can make a very possible and comfortable life for every person with their community. Also, this is very cheap cost wise to buy, and simple in design to troubleshooting themselves. So, the proposed model is identifying and 
Journal of Electrical Engineering and Automation (EEA) (2020)

Vol. 02/ No. 04

Pages: 181-194

https://www.irojournals.com/iroeea

DOI: https://doi.org/10.36548/jeea.2020.4.006

giving feedback very quickly with recognized words. Also, we obtained a real-time recognition rate of $95.6 \%$ with a lot of tests and iteration units which is shown in figure 7.

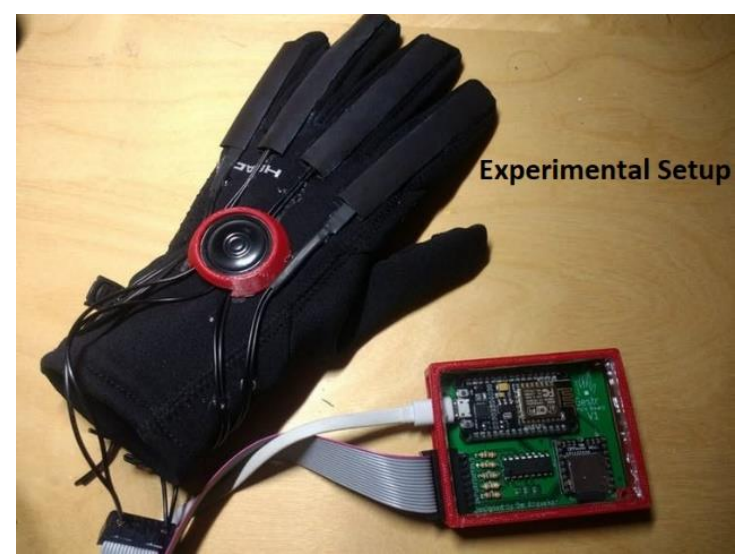

Figure 7 Picture of experimental setup of proposed system

The smart glove is constructed for sign language to text and speech in further development. Their model consists of a bend sensor, microcontroller, communication medium device. This Augmentative and Alternative Communication (AAC) comprises many limitations for this hand gesture language [35][36][37]. The controlling unit is used to control the sensor output and it connects with a smart glove by a microcontroller. The conversion units are incorporated inside the controlling unit. The bend sensor output is converting from voltage to text output for the detection process. Also, there is developed android version devices for communication channel with Bluetooth technology. Here this software application is converting from text into an audible speech signal. The comparison result chart is showing in figure 8.

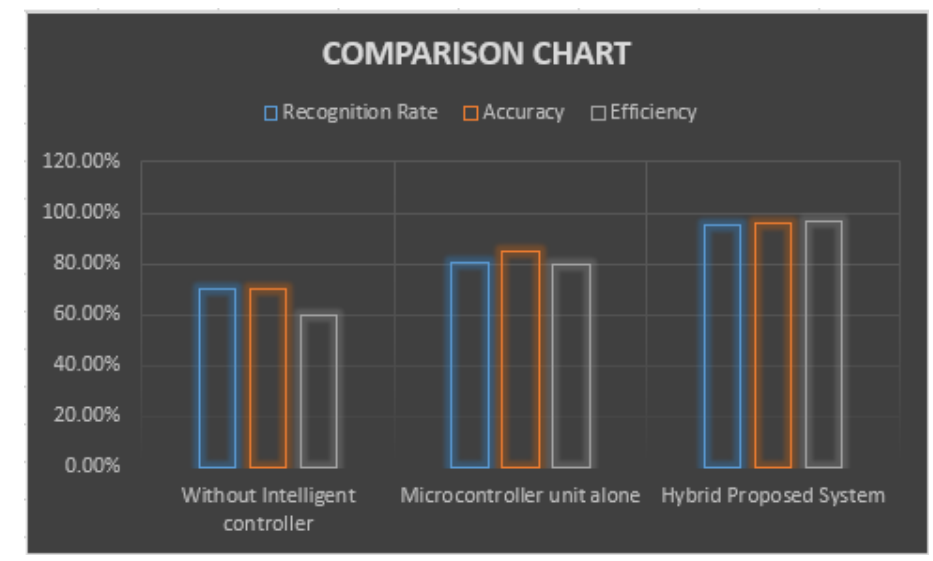

Figure 8 Comparison chart with existing methods 
Journal of Electrical Engineering and Automation (EEA) (2020)

Vol. 02/ No. 04

Pages: 181-194

https://www.irojournals.com/iroeea

DOI: $\underline{\text { https://doi.org/10.36548/jeea.2020.4.006 }}$

The proposing our model consist of many flex sensors to get better results in both the side. A lot of sign symbols are interpreted in the controlling unit. The accuracy level is high based on the specification of the flex sensor. The accurate output of bending action by flex sensor provides a good recognition rate.

\section{CONCLUSION}

Thus our proposed model has been constructed and tested successfully. Our smart glove is used to make a communication medium between deaf and normal people. Also, it is breaking their barrier between them with normal conversation confidently. This glove can spring confidence, comfort in their community. It makes their life's better and carrier can grow. The recognition percentage is also very high compared to another model with various iteration and locality. Our future works are carrying the following phenomena;

1. Implementing artificial neural network in our proposed model.

2. Training many data set leads to a good accuracy level with various parameters and circumstances.

3. Improving speaker quality and deleting or updating the pre-defining dataset.

4. Increasing the number of hand gesture images in the dataset with a machine learning algorithm.

\section{REFERENCES}

[1] Ms. Pallavi Verma, Mrs Shimi S.L, "Design of Smart Gloves", International Journal of Engineering Research and Technology (IJERT) ISSN: 2278-0181. Vol.3 Issue 11, November2014.

[2] M.K. Bhuyan Chaitanya and Darsha Sharath Chandra, "Hand Gesture Animation by Key Frame Extraction”, 2011 International Conference on Image Information Processing (ICIIP 2011), 978-1-61284-861-7/11/\$26.00@2011-IEEE

[3] Praveen Kumar, S Havalagi and Shruthi Urf Nivedita, - The Amazing Gloves that give Voice to the Voicelessl, International Journal of Advances in Engineering \& Technology, Vol. 6, No.1, pp. 471-480, March 2013. 
Journal of Electrical Engineering and Automation (EEA) (2020)

Vol. 02/ No. 04

Pages: 181-194

https://www.irojournals.com/iroeea

DOI: https://doi.org/10.36548/jeea.2020.4.006

[4] Vishal Nayakwadi and N. B. Pokale, -Natural Hand Gestures Recognition System for Intelligent HCI: A Surveyll, International Journal of Computer Applications Technology and Research,Vol.3,No.1,pp. 10 - 19, 2014

[5] Kanika Rastogi, Pankaj Bhardwaj, "A Review Paper on smart glove coverts gestures into speech and text", Moradabad Institute of Technology, International Journal on Recent and Innovation Trends in Computing and Communication .Vol: 4 Issue:5, ISSN: 2321- 8169.

[6] Harmeet Kaur, Amit Saxena, Abhishek Tandon, Keshav Mehrotra and Khushboo Kashyap, "A Review Paper on Evolution of Smart Glove”, International Journal of Scientific Research and Management Studies (IJSRMS), Vol. 3, Issue 3, pp. 124-128, 2016.

[7] K.V.Fale, Akshay Phalke, Pratik Chaudhari and Pradeep Jadhav, "Smart Glove: Gesture Vocalizer for Deaf and Dumb People", International Journal of Innovative Research in Computer and Communication Engineering, Vol. 4, Issue 4, pp. 6800-6806, April 2016.

[8] K.V Fale, Akshay Phalke, Pratik Chaudhari, Pradeep Jadhav, "Smart Glove: Gesture Vocalizer for Deaf and Dump People", JSPM"s Rajarshri Shahu College of Engineering, Pune, India, International Journal of Innovative Research in Computer and Communication Engineering. Vol: 4 Issue: 4, ISSN: 2320-9801.

[9] Sagar P.More and Abdul Sattar, "Hand Gesture Recognition System using Image Processing", International Conference on Electrical, Electronics and Optimization Techniques (ICEEOT), 2016.

[10] Abhishek Tandon, Amit Saxena Keshav Mehrotra, Khushboo Kashyap, Harmeet Kaur, "A Review Paper on Smart Glove - Converts Indian Sign Language (ISL) into Text and Speech", International Journal for Scientific Research \& Development (IJSRD) Vol. 4, Issue 08, pp. 269272, 2016.

[11] Nikhita Praveen, Naveen Karanthand Megha M S, "Sign Language Interpreter Using A Smart Glove", 2014 International conference on Advances in Electronics, Computers and communications (ICAECC). 978-1-4799-5496-1/14/\$31.00 @2014 IEEE

[12] Gunasekaran K. and Maniknandan R., "Sign Language to Speech Translation System Using PIC Microcontroller", International Journal of Engineering and Technology, Vol. 5, No. 2, pp. 1024-1028, 2013. 
Journal of Electrical Engineering and Automation (EEA) (2020)

Vol. 02/ No. 04

Pages: 181-194

https://www.irojournals.com/iroeea

DOI: https://doi.org/10.36548/jeea.2020.4.006

[13] Vajjarapu Lavanya, Akulapravin, M.S., Madhan Mohan, "Hand Gesture Recognition and Voice Conversion System using Sign Language Transcription System", International Journal of Electronics \& Communication Technology, Vol. 5, Issue 4, pp. 145-150, 2014.

[14] Tushar Chouhan, Ankit Panse, Anvesh Kumar Voona and S. M. Sameer, "Smart Glove With Gesture Recognition Ability For The Hearing And Speech Impaired”, IEEE Global Humanitarian Technology Conference - South Asia Satellite (GHTC-SAS), pp. 105-110, September 26-27, 2014. [15] JanFizza Bukhari, Maryam Rehman, Saman Ishtiaq Malik, Awais M. Kamboh and Ahmad Salman, “American Sign Language Translation through Sensory Glove; Sign Speak”, International Journal of uand e-Service, Science and Technology, Vol.8, No.1, pp.131-142, 2015.

[16] Nikhil T. Tarte, Sneha N. Bhadane and Purva S. Kulkarni, "A Gesture Audio Video Conferencing Application for the ease of Communication between Normal Person and Deaf \& Dumb Person", International Journal of Science, Engineering and Technology Research (IJSETR), Vol. 3, Issue 5, pp. 1487-1490, May 2014.

[17] Hussana Johar R.B, Priyanka A, Revathi Amrut M S, Suchitha K, Sumana K J "Multiple sign language translation into voice", International Journal of Engineering and Innovative Technology (IJEIT), Vol. 3, Issue 10, April 2014

[18] Abjhijit auti, V. G. Puranik, Dr. A.K. Kureshi, "Speaking gloves For Speechless Persons", IJIRSET, Volume 3, Issue 4, April 2014, PP. 282-290.

[19] M. P. Paulraj, S. Yaacob, H. Desa, and W. Majid, "Gesture Recognition System for Kod Tangan Bahasa Melayu (KTBM) using Neural Network", in 5th International Colloquium on Signal Processing and Its Applications (CSPA), pp. 19-22, 2009.

[20] M. Mohandes, S. I. Quadri, and M. D. King, "Arabic Sign Language Recognition an Image Based Approack", in 21st International Conference on Advanced Information Networking and Applications Workshops, 2007 (AINAW'07), pp. 272-276, 2007.

[21] S. J. Wang, D. C. Zhang, C. C. Jia, N. Zhang, C. G. Zhou, and L. B. Zhang, “A Sign Language Recognition Based on Tensor", in Second International Conference on Multimedia and Information Technology (MMIT), pp. 192-195, 2010.

[22] Kuo Chue Neo, Haidi Ibrahm and Wan Mohd Yusof Rahiman Wan Abdul Aziz, "Development of Sign Signal Translation System Based on Altera's FPGA DE2 Board", International Journal of Human Computer Interaction (IJHCI), Vol. 2, Issue 3, pp. 101-114, 2011. 
Journal of Electrical Engineering and Automation (EEA) (2020)

Vol. 02/ No. 04

Pages: 181-194

https://www.irojournals.com/iroeea

DOI: https://doi.org/10.36548/jeea.2020.4.006

[23] K. Park, J. H. Kim, and K. S. Hong, "An Implementation of an FPGA-Based Embedded Gesture Recognizer using a Data Glove", in Proceedings of the 2nd International Conference on Ubiquitous Information Management and Communication (ICUIMC'08), 2008.

[24] W. K. Chung, W. Xinyu, and Y. Xu, "A Real-time Hand Gesture Recognition Based on Haar Wavelet Representation", in Proceedings of the 2008 IEEE International Conference on Robotics and Biomimetics, Washington, DC, USA, pp. 336-341, 2008.

[25] Taner Arsan and Oğuz Ülgen, "Sign Language Converter”, International Journal of Computer Science \& Engineering Survey (IJCSES), Vol. 6, No.4, pp. 39-51, August 2015.

[26] Sachin Bhat, Amruthesh M, Ashik, Chidanand Das and Sujith, "Translating Indian Sign Language to text and voice messages using flex sensors", International Journal of Advanced Research in Computer and Communication Engineering, Vol. 4, Issue 5, pp. 430- 434, May 2015. [27] Rohit Rastogi, Shashank Mittal and Sajan Agarwal, "A Novel Approach for Communication among Blind, Deaf and Dumb People”, 2015 2nd International Conference on Computing for Sustainable Global Development (INDIA Com), 978-9-3805-4416- 8/15/\$31.00 @2015 IEEE [28] JanFizza Bukhari, Maryam Rehman, Saman Ishtiaq Malik, Awais M. Kamboli and Ahmad Salman "American Sign Language Translation through Sensory Glove Sign Speak "2015 International Journal of $U$ and E Service, Science and Technology National University of Science and Technology, Islamabad, Pakistan.

[29] Vajjarapu Lavanya, Akulapravin, M.S. Madhan Mohan "Hand Gesture Recognition And Voice Conversion System Using Sign Language Transcription System” 2014 International Journal of Electronics and Communication Engineering BVC Engineering College, Odalarevu AP, India. [30] Sankar kumar, Jenitha J, Narmadha, Suganyaa, "An embedded Module as virtual tongue for voiceless", Volume 4, Issue.3, May 2014, PP. 155-163.

[31] Syed faiz Ahmed, Syed Muhammad Baber Ali, Sh. Saqib Munawwar Qureshi, "Electronics speaking Gloves for Speechless patients", IEEE, Nov 2010, PP. 56-60.

[32] Yun Li,Xiang Chen, Jianxun Tian, Xu Zhang, Kongqiao Wang and Jihai Yang, -Automati Recognition of Sign Language Subwords based on Portable Accelerometer and EMG Sensorsll, IEEE Engineering in Medicine and Biology Society, Vol.59, No.10, pp. 2695 -2704, October 2012. 
Journal of Electrical Engineering and Automation (EEA) (2020)

Vol. 02/ No. 04

Pages: 181-194

https://www.irojournals.com/iroeea

DOI: https://doi.org/10.36548/jeea.2020.4.006

[33] Praveen kumar , S. Havalag, Shruthi, Urf Nivedita, "The Amazing Digital Gloves that give voice to the voiceless", IJAET, Volume 6, Issue 1, March 2013, PP. 471-480.

[34] S. J. Wang, D. C. Zhang, C. C. Jia, N. Zhang, C. G. Zhou, and L. B. Zhang, "A Sign Language Recognition Based on Tensor", in Second International Conference on Multimedia and Information Technology (MMIT), pp. 192-195, 2010.

[35] Kuo Chue Neo, Haidi Ibrahm and Wan Mohd Yusof Rahiman Wan Abdul Aziz, "Development of Sign Signal Translation System Based on Altera's FPGA DE2 Board", International Journal of Human Computer Interaction (IJHCI), Vol. 2, Issue 3, pp. 101-114, 2011. [36] Solanki Krunal M, "Indian Sign Languages using Flex Sensor Glove”, International Journal of Engineering Trends and Technology (IJETT), Vol. 4, Issue6, June 2013.

[37] Anbarasi Rajamohan, Hemavathy R., Dhanalakshmi M., "Deaf-Mute Communication Interpreter", International Journal of Scientific Engineering and Technology, Vol. 2, Issue 5, pp. 336-341, 2013. 